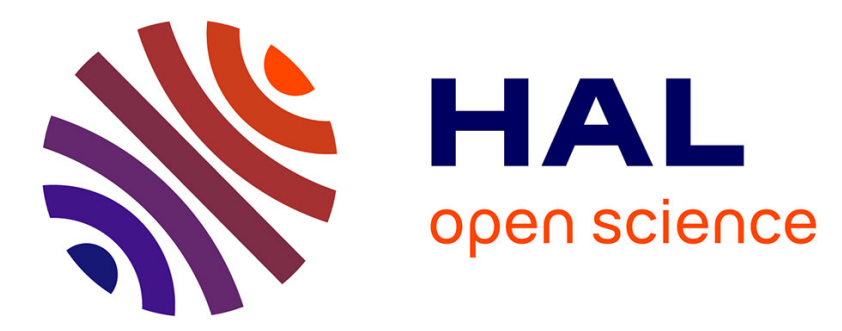

\title{
Electrical Spin Injection into the 2D Electron Gas in AlN/GaN Heterostructures with Ultrathin AlN Tunnel Barrier
}

Xiaoyue N Zhang, Ning Tang, Liuyun Yang, Chi Fang, Caihua Wan, Xingchen Liu, Shixiong Zhang, Yunfan Zhang, Xinqiang Wang, Yuan Lu, et al.

\section{To cite this version:}

Xiaoyue N Zhang, Ning Tang, Liuyun Yang, Chi Fang, Caihua Wan, et al.. Electrical Spin Injection into the 2D Electron Gas in AlN/GaN Heterostructures with Ultrathin AlN Tunnel Barrier. Advanced Functional Materials, 2021, 31 (15), pp.2009771. 10.1002/adfm.202009771 . hal-03417701

\section{HAL Id: hal-03417701 https://hal.science/hal-03417701}

Submitted on 10 Nov 2021

HAL is a multi-disciplinary open access archive for the deposit and dissemination of scientific research documents, whether they are published or not. The documents may come from teaching and research institutions in France or abroad, or from public or private research centers.
L'archive ouverte pluridisciplinaire HAL, est destinée au dépôt et à la diffusion de documents scientifiques de niveau recherche, publiés ou non, émanant des établissements d'enseignement et de recherche français ou étrangers, des laboratoires publics ou privés. 


\title{
Electrical spin injection into the two-dimensional electron gas in AIN/GaN heterostructures with ultra-thin AIN barrier
}

\author{
Xiaoyue Zhang ${ }^{a}$, Ning Tang ${ }^{\star}{ }^{a d}$, Liuyun Yang ${ }^{a}$, Chi Fang ${ }^{b}$, Caihua Wan ${ }^{b}$, Xingchen
}

Liu $^{a}$, Shixiong Zhang ${ }^{a}$, Yunfan Zhang ${ }^{a}$, Xinqiang Wang, Yuan Luc, Weikun Gea, Xiufeng $\operatorname{Han}^{b}$ and Bo Shen*

${ }^{\circledR}$ State Key Laboratory of Artificial Microstructure and Mesoscopic Physics, School of Physics, Peking University, Beijing 100871, China. E-mail: ntang@pku.edu.cn,

\section{bshen@pku.edu.cn}

${ }^{b}$ Beijing National Laboratory for Condensed Matter Physics, Institute of Physics, University of Chinese Academy of Sciences, Chinese Academy of Sciences, Beijing 100190, China. 'Institut Jean Lamour, Université de Lorraine, CNRS UMR7198, Campus ARTEM, 2 Allée André Guinier, BP 50840, 54011 Nancy, France

${ }^{d}$ Frontiers Science Center for Nano-optoelectronics \& Collaboration Innovation Center of Quantum Matter, Peking University, Beijing 100871, China

\begin{abstract}
:
Spin injection into the two-dimensional electron gas (2DEG) in AlN/GaN heterostructures was studied by magneto-transport measurements. An ultra-thin AlN layer at the hetero-interface acts as a barrier to form 2DEG in the triangular GaN
\end{abstract}


quantum well and a tunneling barrier for the spin injection to overcome spin conductance mismatch issue. Hanle signals and inversed Hanle signals were observed, proving that the spin injection was achieved in the 2DEG in the AlN/GaN heterostructure rather than in the interfacial states. The spin relaxation time in $2 \mathrm{DEG}$ at $8 \mathrm{~K}$ was found as long as $850 \mathrm{ps}$, which almost keeps constant with bias and decreases with increasing temperature. The spin relaxation process can be explained by D’yakonov Perel mechanisms originated from Rashba spin orbit coupling. These results show the promising potential of $2 \mathrm{DEG} \mathrm{AlN} / \mathrm{GaN}$ heterostructures for the spin field effect transistor applications.

Semiconductor spintronics, focusing on the electron's spin degree of freedom, has been a major interest in recent researches for the application of high performance and low energy consumption devices. Spin field effect transistor (SFET) is a representative spintronic devices, ${ }^{1,2}$ offering potential advantages over classical transistor driven by the charge of the electrons. The core issues of SFET can be generally concluded as three parts: spin injection, ${ }^{3,4}$ manipulation ${ }^{5,6}$ and detection. ${ }^{7,8} \mathrm{GaN}$ based material has attracted a major interest in semiconductor spintronics for its promising applications at room temperature. ${ }^{9,10,11}$ Two-dimensional electron gas (2DEG) in GaN-based heterostructures is widely used in electronic devices for its excellent electrical properties, such as high electron concentration and mobility. Besides, a large spin relaxation time is expected. Theoretical calculation figures out that the spin orbit coupling (SOC) can be minimized with specific band structure in GaN-based 
2DEG. ${ }^{12,13}$ Meanwhile, 2DEG in GaN-based heterostructures may provide more opportunity for the realization of spin manipulation with tunable SOC. ${ }^{14,15}$ However, up to now, the complete Datta \& Das model SFET has only been realized in InGaAsbased 2DEG. One is based on Ferromagnetic(FM)/insulator tunneling junction ${ }^{16}$ while the other is based on quantum point contact. ${ }^{17}$ For GaN based materials, electrical spin injection and detection has not yet been realized in the $2 \mathrm{DEG} \cdot{ }^{18,19}$ A key challenge is the spin injection in 2DEG in GaN based heterostructures.

$\mathrm{FM} /$ insulator junction is commonly used as an injector to realize efficient spin injection. ${ }^{20,21}$ The main obstacle to apply it on $2 \mathrm{DEG}$ is the large conductance mismatch between FM and the barrier of the 2DEG, and extra spin dephasing in the barrier. As for $\mathrm{GaN}$ based material, $\mathrm{AlGaN} / \mathrm{GaN}$ is the most mature and representative structure in forming $2 \mathrm{DEG},{ }^{22,23}$ while the $\mathrm{AlGaN}$ thickness is at least $3.5 \mathrm{~nm}$ in order to form $2 \mathrm{DEG}^{24}$, and for high performance $2 \mathrm{DEG}$ the thickness is about $15-25 \mathrm{~nm} .{ }^{25}$ Thus, with insulator/AlGaN structure, the thick $\mathrm{AlGaN}$ layer hampers spin injection by causing spin dephasing before being injected into 2DEG. It also results in quite small junction conductance and thus large conductance mismatch. With a sole $\mathrm{AlGaN}$ barrier, its large thickness and low barrier height make it hard for electron tunneling. In this study, an in-situ grown ultra-thin AIN layer is used as the barrier of the 2DEG triangle quantum well as well as tunnel barrier to overcome conductance mismatch simultaneously. Fig. 1 illustrate this structure and the spin injection process. For testing the effect of such an ultra-thin AlN layer, we fabricated a non-local three terminal (3T) device with AlN/GaN heterostructure. Hanle spin precession was observed in the $3 \mathrm{~T}$ device of the $2 \mathrm{DEG}$ in 
AlN/GaN heterostructures at various temperatures and biases. Spin dynamics is also studied based on 3T Hanle measurements.

\section{Results}

\section{Device configuration and the electrical transport parameters}

Fig. 2(a) shows the schematic of the $3 \mathrm{~T}$ device. The fabrication process is described in Methods. All the three electrodes consist of $\mathrm{Au} / \mathrm{Co} / \mathrm{AlN} / \mathrm{GaN}$. The two electrodes on both sides are reference electrodes, while the middle one acts as the spin injector. Fig. 2(b) shows a typical IV characterization of the Co/AIN/2DEG contact. The non-linear dependence unveiled the tunneling process in the junction below $55 \mathrm{~K}$. Above $55 \mathrm{~K}$, the IV curves exhibit linear ohmic contact behavior. Tunneling is hardly observed for the thermal energy is sufficiently high. For further illustration of the AlN tunneling properties, zero-bias resistance (ZBR) was measured as a function of temperature. A weak dependence of the zero-bias resistance shown in Fig. 2(c) indicates a one-step tunneling of $\mathrm{Co} / \mathrm{AlN} / \mathrm{GaN}$ heterostructures at temperature lower than $55 \mathrm{~K}$.

Magneto-transport measurements were conducted with van der Pauw geometry. From the Hall measurements, the electron density is determined to be $n_{e 1}=2.2 \times 10^{13} \mathrm{~cm}^{-2}$ and the corresponding mobility is $\mu=480 \mathrm{~cm}^{2} V^{-1} \mathrm{~s}^{-1}$ at $2 \mathrm{~K}$. The Shubnikov-de Haas ( $\mathrm{SdH})$ oscillations were observed, as shown in the inset of Fig. 2(d), indicating an even higher mobility compared with the Hall mobility. In the inset of Fig. 2(d), the SdH oscillations are caused only by the perpendicular component $B_{z} \cos \theta$ of the magnetic field, which demonstrates the 2D characteristic of the electrons. The oscillation frequency is extracted to be $14.9 \mathrm{~T}$. The corresponding electron density is then 
$n_{e 2}=7.2 \times 10^{11} \mathrm{~cm}^{-2} \cdot 26,27$ The difference in the electron densities determined by Hall and $\mathrm{SdH}$ respectively indicates the occupancy of at least two sub-bands in the triangular quantum well at $2 \mathrm{~K}$, while the $\mathrm{SdH}$ oscillation is only induced by the excited state. The different mobility suggests the strong scattering mainly dominates at the AlN/GaN interface. In conclusion, the first sub-band is fully occupied with electron density two orders higher and the electrons are strongly scattered at the interface.

\section{T Hanle measurements}

3T Hanle measurements were performed as schematically shown in Fig. 2(a). A constant current is injected from the middle spin injection electrode to one reference electrode, while the voltage is measured between the spin injector and the other electrode. Spin accumulation can be detected with Hanle effect by applying a perpendicular magnetic field $B_{z}$. Electron spins injected via the tunnel junction into the 2DEG precess at a rate $\omega_{L}=\mathrm{g} \mu B_{z} / \hbar \quad(\mathrm{g}=2)^{28,29}$, while they also dephase at the same time. The spin-induced electrochemical potential in the 2DEG changes with increasing magnetic field. Sweeping the perpendicular magnetic field gives a Lorentz-shaped magneto-resistance corresponding to Hanle precession as shown in Fig. 3(a). Considering the electrode width is $20 \mu \mathrm{m}$, which is much larger than the spin diffusion length, ${ }^{18}$ the measured voltage between injector and reference electrode can be approximated as $\mathrm{V}_{3 T}\left(B_{z}\right)=\mathrm{V}_{3 T}(0) /\left[1+\left(\omega_{L} \tau_{s}\right)^{2}\right]$, where $\tau_{s}$ is spin lifetime and $\omega_{L}$ is the spin precession rate. ${ }^{30} \mathrm{~V}_{3 T}(0)$ is the spin accumulation voltage. The fitting curve is shown as the black line in Fig. 3(a). The extracted spin relaxation time is $573.0 \pm 21$ ps at $55 \mathrm{~K}$. 
The inverted Hanle signal was observed in the same device with parallel magnetic field $B_{\square}$, as shown in Fig. 3(a). The inverted Hanle signal is commonly recognized as spin-dependent signal caused by rough interface. ${ }^{31}$ Variously oriented stray field from rough $\mathrm{Co} / \mathrm{AlN}$ interface induces random spin precession and thus enhances spin relaxation under zero external magnetic field. With increasing parallel magnetic field, random precession is reduced and spin is gradually polarized along $B_{\square}$ in 2DEG. Highly polarized spins give rise to a larger electrochemical potential than the dephased spins, which induces a minimum voltage at $B_{\square}=0$. The coexistence of Hanle and inverted Hanle signal in the same device guarantees this Lorentzian-shaped 3T signal from spin accumulation instead of other quantum effects ${ }^{31}$ or interfacial influences. ${ }^{32,33}$ The inverted Hanle signal $(\Delta \mathrm{V}=7.6 \mu \mathrm{V})$ is slight smaller than the Hanle signal $(\Delta \mathrm{V}=$ $10.5 \mu \mathrm{V}$ ), indicating the ignorable influence of local static magnetic field due to the rough $\mathrm{Co} / \mathrm{AlN}$ interface. Since the unintentionally doped GaN cannot serve as a transport channel with small conductivity ${ }^{34}$, the observed spin accumulation mainly occurs in the $2 \mathrm{DEG}$ rather than the bulk GaN.

\section{Bias dependence of the Hanle signal}

Comparison of detailed measurements of Hanle and inverted Hanle signals indicates the different origins and excludes confusing signals from $\mathrm{Co}$ or $\mathrm{Co} / \mathrm{AlN}$ interface. As shown in Fig. 3(b), the amplitude of Hanle signal is weakly decreased with increasing bias. However, with magnetic field parallel to the surface, the corresponding inverted Hanle signal is almost constant, as shown in Fig. 3(c). It figures out the Hanle signal is originated from spin accumulation in semiconductor. The amplitude of spin 
accumulation is dependent on injection current. Whereas the inverted Hanle signal is commonly recognized as cobalt induced stray field being compensated with $B_{\square} \cdot 35,3$ With different bias, inverted Hanle signals are almost stable. Amplitude and orientation of the stray field stays constant with different bias. Corresponding to the amplitude of the stray field, the full width at half maximum (FWHM) of the inverted Hanle signals stays almost constant as $50 \mathrm{Oe}$. And the FWHM of the Hanle signals is around $150 \mathrm{Oe}$, which is three times larger and also points to the difference of Hanle and inverted Hanle signals. It further excludes artifacts such as broad Hanle signals from dead layer or quantum signals induced by Co/AlN interface. ${ }^{3}$ Here, $2^{\text {nd }}$ harmonic measurements were conducted for clearer Hanle signal without background signals. The $2^{\text {nd }}$ harmonic signals show exact the same behavior as $1^{\text {st }}$ harmonic signals in our experiments and in Ref. 36.

For the $3 \mathrm{~T}$ Hanle measurements, spin injector and detector share one electrode. Spin in the interfacial states can be measured ${ }^{33}$ but confused with spin in 2DEG. To distinguish them, signals from the interfacial states can be easily tuned by bias caused band bending and thus separated from the 2DEG signals. Careful measurements with different amplitudes of AC were conducted. Fig. 3(b) shows the Hanle signals with various bias at $35 \mathrm{~K}$. Characterized by the FWHM, $\tau_{s}$ shows weak dependence on bias. Further study is shown in Fig. 3(d), where the spin relaxation time was extracted for different bias at different temperatures. For each bias, the temperature dependence is almost the same, as $\tau_{s}$ decreasing with increasing temperature. At each temperature, spin relaxation time shows similar weak dependence on bias. Signals from localized 
states are thus ruled out. ${ }^{33}$

\section{Temperatrue dependence of the Hanle signal}

The dependence of the Hanle signal on temperature is also measured in the range of 8-45 K. Fig. 4(a) and 4(c) show the temperature dependent behaviors for Hanle and inverted Hanle signals, respectively. is also observed in. The spin relaxation time was then extracted by fitting the Hanle curves, which is shown in Fig. 4(b). In Figs. 4(a) and 4(b), with increasing temperature, the spin relaxation time decreases. The maximum spin relaxation time corresponds to 860 ps at $8 \mathrm{~K}$. It is also observed that the Hanle signals' amplitude decreases rapidly with increasing temperature. As shown in Fig. 2(b), the tunneling barrier reduces as temperature increases,. Since spin flips are enhanced with smaller interface tunneling resistance, the enhanced spin flipping causes decreasing of the Hanle signals with increasing temperature. According to previous experiments and theoretical analysis, D'yakonov Perel' (DP) mechanism is the dominant mechanism in wurtzite $\mathrm{GaN}$ based materials. ${ }^{18,37}$ Calculations are made to check if the DP mechanism fits our experiments. The fitting is shown as the red curve in Fig. 4(b). For the DP mechanism, the spin relaxation time is expressed as $1 / \tau_{s} \propto \tau_{P}\left(A T-B T^{2}+C T^{3}\right)$. The linear-to-temperature term represents the Rashba term, the parabolic is the interference term, and the cubic term is the Dresselhaus term, where $\mathrm{A}, \mathrm{B}$ and $\mathrm{C}$ are parameters related with material structures and are constant at various temperatures. The Dresselhaus SOC coefficient can be calculated with formula $\beta=b\left(\gamma\left\langle k_{z}\right\rangle^{2}-k_{\square}^{2}\right)$. For 2DEG in AlN/GaN heterostructures, the Dresselhaus SOC parameter is estimated to be the same as that in bulk GaN, $b \approx 4, \quad \gamma=0.33 \mathrm{eVA}^{\circ} .^{37}$ 
Suppose triangle well width to be $5 \mathrm{~nm}$, then $\left\langle k_{z}\right\rangle^{2} \square(\pi / 5 \mathrm{~nm})^{2}$. And $k_{\square}^{2}$ can be replaced with the Fermi wavevector $k_{F}^{2}=2 \pi n_{e}$. Considering the very shallow $2^{\text {nd }}$ subband (E2) occupancy, $k_{\square}^{2}$ is a little overestimated, but the overestimation is ignorable with two orders difference of electron concentration between the two sub-bands. With a large $n_{e}$ in our 2DEG structures, the Dresselhaus SOC is calculated to be almost zero and may even offset a small portion of the Rashba SOC, corresponding to neglectable $\mathrm{B}$ and $\mathrm{C}$ calculated in Fig. 4(b). The experimental results fit well with previous theoretical calculations of $2 \mathrm{DEG}$ rather than bulk GaN. ${ }^{18}$ The elimination of the Dresselhaus SOC offers a probability of ultra-long spin relaxation time in 2DEG AlN/GaN heterostructures, because Rashba term is tunable with gate while Dresselhaus is inaccessible.

In conclusion, spin injection into the $2 \mathrm{DEG}$ in $\mathrm{AlN} / \mathrm{GaN}$ heterostructures is realized with an ultra-thin AlN tunnel barrier which also acts as the potential barrier to form 2DEG. The Hanle signal is observed up to $45 \mathrm{~K}$, and the maximum spin relaxation time is about 860 ps at $8 \mathrm{~K}$. The DP mechanism is illustrated to dominate the spin relaxation with mainly Rashba SOC while the Dresselhaus SOC is neglectable. This work shows that spin injection with AIN barrier into 2DEG provides a way to realize integrated SFET in 2DEG in GaN based heterostructures system.

\section{Methods}

AlN/GaN heterostructures were grown epitaxially by molecular beam epitaxy (MBE) on the GaN template. A $120 \mathrm{~nm}$ thick layer of unintentionally doped $\mathrm{GaN}$ was 
grown, followed by a $2.5 \mathrm{~nm}$ layer of AlN. ${ }^{38,39}$ Then the photoresist was spin-coated on the $\mathrm{AlN} / \mathrm{GaN}$ heterostructures to prevent oxidation of the $\mathrm{AlN}$ as soon as possible. Device fabrication was initiated with standard ultraviolet lithography. After 40-second etching with Ar ion beam to fully remove aluminum oxides at the surface, a $20 \mathrm{~nm} \mathrm{Co}$ and a $5 \mathrm{~nm} \mathrm{Au}$ were deposited subsequently with e-beam evaporation in situ. An injector with dimension of $20 \times 200 \mu \mathrm{m}^{2}$ was completed after lift-off. Also, two reference contacts were fabricated with dimension of $150 \times 200 \mu \mathrm{m}^{2}$. The distance between two nearby electrodes is about $230 \mu \mathrm{m}$, far more than the spin diffusion length in GaN. The schematic of the heterostructures and the dimensions of each contact are shown clearly in Fig. 2(a). The electrical measurements were performed in a physics parameter measurement system (PPMS), with temperature range of 2-300 K. Keithley 6221 is used for applying alternative current (AC). A lock-in amplifier (Stanford Research SR830) is used for spin detection. Meanwhile, Hall measurements and IV characterization are conducted with Keithley 2400.

\section{References:}

1. Datta, S. \& Das, B. Electronic analog of the electro-optic modulator. Appl. Phys. Lett. 56, 665 (1990). 
2. Schliemann, J., Egues, J. C. \& Loss, D. Nonballistic Spin-Field-Effect Transistor. Phys. Rev. Lett. 90, 146801 (2003).

3. Sato, S., Nakane, R., Hada, T. \& Tanaka, M. Spin injection into silicon in three-terminal vertical and four-terminal lateral devices with $\mathrm{Fe} / \mathrm{Mg} / \mathrm{MgO} / \mathrm{Si}$ tunnel junctions having an ultrathin Mg insertion layer. Phys. Rev. B. 96, 235204 (2017).

4. Tahara, T. et al. Observation of large spin accumulation voltages in nondegenerate Si spin devices due to spin drift effect: Experiments and theory. Phys. Rev. B 93, 214406 (2016).

5. Yan, W. et al. A two-dimensional spin field-effect switch. Nat. Commun. 7, 13372 (2016).

6. Chuang, P. et al. All-electric all-semiconductor spin field-effect transistors. Nat. Nanotechnol. 10, 35-39 (2014).

7. Oltscher, M. et al. Gate-tunable large magnetoresistance in an all- semiconductor spin valve device. Nat. Commun. 8, 1807 (2017).

8. Park, T.-E. et al. Large spin accumulation and crystallographic dependence of spin transport in single crystal gallium nitride nanowires. Nat. Commun. 8, 15722 (2017).

9. Diode, S. L. E., Bhattacharya, A., Member, G. S. \& Baten, Z. Room Temperature GaN-Based Edge-Emitting. 29, 338-341 (2017).

10. Chen, J.-Y., Wong, T.-M., Chang, C.-W., Dong, C.-Y. \& Chen, Y.-F. Self-polarized spinnanolasers. Nat. Nanotechnol. 9, 845-850 (2014).

11. Hu, C. Y. et al. Spin precession of holes in wurtzite GaN studied using the time-resolved Kerr rotation technique. Phys. Rev. B 72, 121203 (2005).

12. Harmon, N. J., Putikka, W. O. \& Joynt, R. Prediction of extremely long mobile electron spin lifetimes at room temperature in wurtzite semiconductor quantum wells. Appl. Phys. Lett. 98, 
073108 (2011).

13. Fu, J. et al. Spin-orbit coupling in wurtzite heterostructures. Phys. Rev. B 101, 134416 (2020).

14. Liu, X. et al. Effective Manipulation of Spin Dynamics by Polarization Electric Field in InGaN/GaN Quantum Wells at Room Temperature. Adv. Sci. 7, 1903400 (2020).

15. Yin, C. et al. Rashba and Dresselhaus spin-orbit coupling in GaN-based heterostructures probed by the circular photogalvanic effect under uniaxial strain. Appl. Phys. Lett. 97, 181904 (2010).

16. Koo, H. C. et al. Control of spin precession in a spin-injected field effect transistor. Science 325, 1515-1518 (2009).

17. Chuang, P. et al. All-electric all-semiconductor spin field-effect transistors. Nat. Nanotechnol. 10, 35-39 (2014).

18. Jahangir, S., Doğan, F., Kum, H., Manchon, A. \& Bhattacharya, P. Spin diffusion in bulk GaN measured with MnAs spin injector. Phys. Rev. B. 86, 035315 (2012).

19. Bhattacharya, A., Baten, M. Z. \& Bhattacharya, P. Electrical spin injection and detection of spin precession in room temperature bulk GaN lateral spin valves. Appl. Phys. Lett. 108, 042406 (2016).

20. Fert, A. \& Jaffre, H. Conditions for efficient spin injection from a ferromagnetic metal into a semiconductor. Phys. Rev. B. 64, 184420 (2001).

21. Parkin, S. S. P. et al. Giant tunnelling magnetoresistance at room temperature with $\mathrm{MgO}$ ( 100 ) tunnel barriers. Nat. Mater. 3, 862-867 (2004).

22. Mazumder, B. et al. Atom probe analysis of AlN interlayers in AlGaN/AlN/GaN heterostructures. Appl. Phys. Lett. 102, 111603 (2013). 
23. Tilak, V. et al. Influence of barrier thickness on the high-power performance of AlGaN/GaN HEMTs. IEEE Electron Device Lett. 22, 504-506 (2001).

24. Ibbetson, J. P. et al. Polarization effects, surface states, and the source of electrons in AlGaN / GaN heterostructure field effect transistors. Appl. Phys. Lett. 77, 250 (2000).

25. Liu, G. et al. A theoretical calculation of the impact of GaN cap and $\mathrm{Al} \times \mathrm{Ga} 1-\mathrm{xN}$ barrier thickness fluctuations on two-dimensional electron gas in a GaN/AlxGa1-xN/GaN heterostructure. IEEE Trans. Electron Devices 58, 4272-4275 (2011).

26. Cao, Y., Wang, K., Orlov, A., Xing, H. \& Jena, D. Very low sheet resistance and Shubnikovde-Haas oscillations in two-dimensional electron gases at ultrathin binary AlNGaN heterojunctions. Appl. Phys. Lett. 92, 152112 (2008).

27. Zheng, Z. W. et al. Transport properties of two-dimensional electron gas in different subbands in triangular quantum wells at AlxGa1-xN/GaN heterointerfaces. Appl. Phys. Lett. 82, 1872 (2003).

28. Shchepetilnikov, A. V. et al. Electron spin resonance in a 2D system at a GaN/AlGaN heterojunction. Appl. Phys. Lett. 113, 052102 (2018).

29. Frayssinet, E., Maude, D. K., Falko, V. \& Khan, A. Effective g* factor of two-dimensional electrons in GaN/AlGaN heterojunctions. Appl. Phys. Lett. 75, 3156 (1999).

30. Dash, S. P. et al. Spin precession and inverted Hanle effect in a semiconductor near a finiteroughness ferromagnetic interface. Phys. Rev. B. 84, 054410 (2011).

31. Txoperena, O. et al. Impurity-Assisted Tunneling Magnetoresistance under a Weak Magnetic Field. Phys. Rev. Lett. 113, 146601 (2014).

32. Wan, C. H. et al. Nonlocal magnetoresistance due to Lorentz force in linear transport region in 
bulk silicon. Appl. Phys. Lett. 103, 262406 (2013).

33. Tran, M. et al. Enhancement of the spin accumulation at the interface between a spin-polarized tunnel junction and a semiconductor. Phys. Rev. Lett. 102, 036601 (2009).

34. Zube, C. et al. Spin injection in epitaxial $\mathrm{MnGa}(111) / \mathrm{GaN}(0001)$ heterostructures. J. Appl. Phys. 123, 033906 (2018).

35. Aoki, Y. et al. Investigation of the inverted Hanle effect in highly doped Si. Phys. Rev. B. 86, 081201 (2012).

36. Fang, C. et al. Determination of spin relaxation times in heavy metals via second-harmonic spin injection magnetoresistance. Phys. Rev. B. 96, 134421 (2017).

37. Bu, J. H., Rudolph, J., Natali, F., Semond, F. \& Hägele, D. Temperature dependence of electron spin relaxation in bulk GaN. Phys. Rev. B. 81, 155216 (2010).

38. Yang, L. et al. Planar anisotropic Shubnikov-de-Haas oscillations of two-dimensional electron gas in AlN/GaN heterostructure. Appl. Phys. Lett. 115, 152107 (2019).

39. Yang, L. et al. Three Subband Occupation of the Two-Dimensional Electron Gas in Ultrathin Barrier AlN/GaN Heterostructures. Adv. Funct. Mater. 2004450, 1-7 (2020). 


\section{Acknowledgements}

This work was supported by National Key Research and Development Program of China (Nos. 2018YFB0406603, and 2018YFE0125700), the National Natural Science Foundation of China (Nos. 61927806, 61574006, 61522401, 61521004, and 11634002). Y.L. acknowledges the support from the French National Research Agency (ANR) FEOrgSpin project (Grants No. ANR-18-CE240017-01) and also ICEEL BlueSpinLED and SHATIPN projects.

\section{Author contributions}

N. T. conceived the study. L.Y. conducted the MBE growth of the samples. X.

Z. and X.L. performed the fabrication and measurements of the device. X. Z., N. T., L. Y., C. F., C. W., Y. L. and W. G. analyzed the data, and wrote the manuscript. All the authors contributed to the scientific discussion and manuscript revision. N.T. and B. S. co-supervised the work.

\section{Conflict of interest}

The authors declare no conflict of interest. 


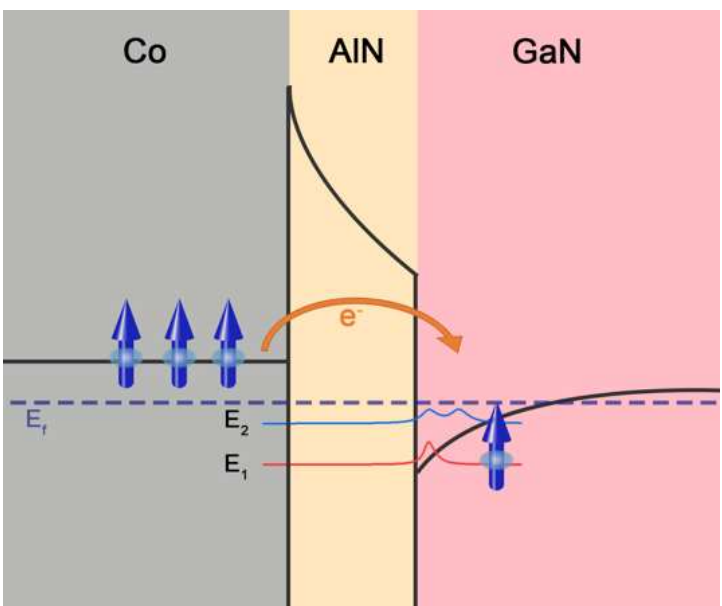

Figure 1. Illustration of electron spin injection with $\mathrm{Co} / \mathrm{AlN} / \mathrm{GaN}$ structure. $\mathrm{E}_{\mathrm{f}}$ refers to Fermi level. $E_{1}$ and $E_{2}$ are schematic wave functions of the two sub-bands existing in the $\mathrm{AlN} / \mathrm{GaN}$ heterostructure. 
a

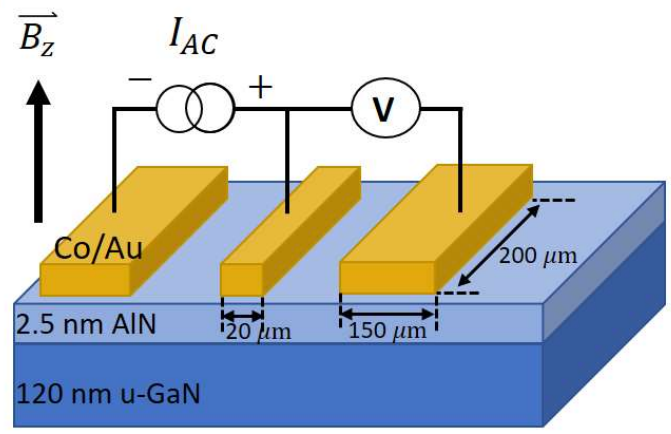

C

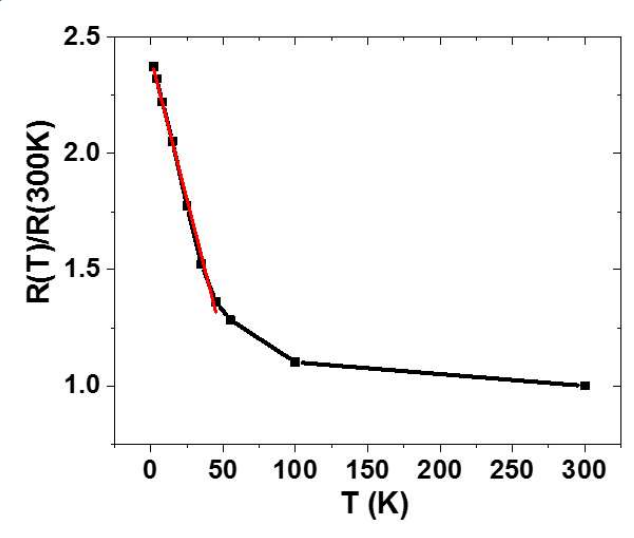

b

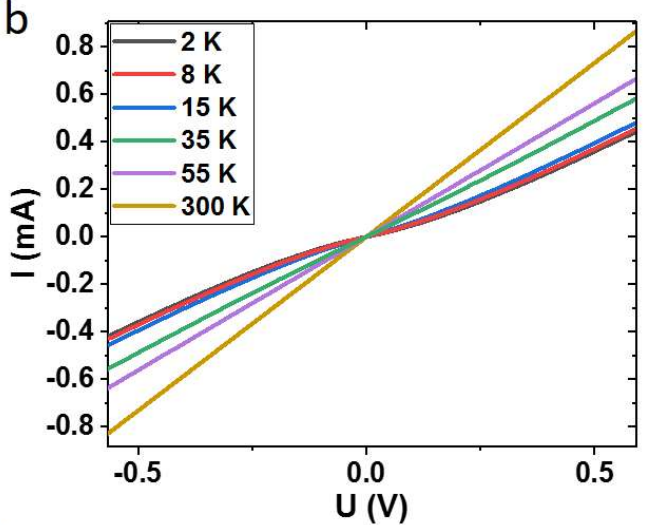

d

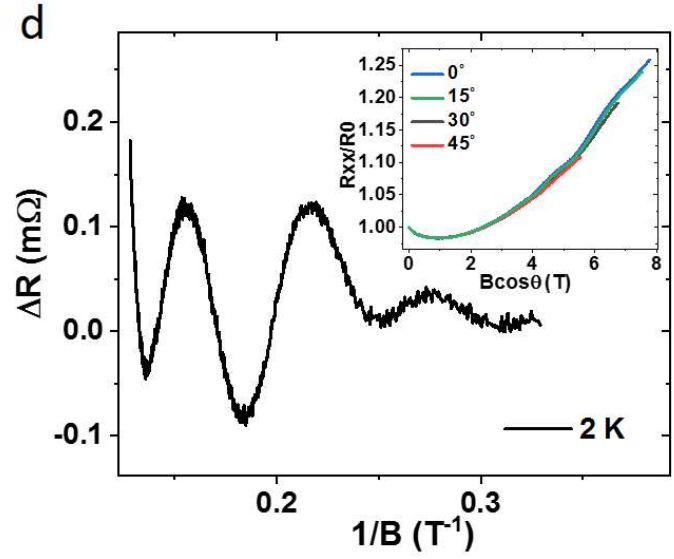

Figure 2. (a) Device schematic and configuration of $3 \mathrm{~T}$ Hanle measurement. (b) I-V characteristic of two Co/AlN/GaN contact in 2- $300 \mathrm{~K}$. (c) Zero-bias resistance as a function of temperature. (d) Magnitude of $\mathrm{SdH}$ oscillations as a function of perpendicular magnetic field 1/B at $2 \mathrm{~K}$. Inset shows longitudinal magnetoresistance as a function of perpendicular magnetic field. 

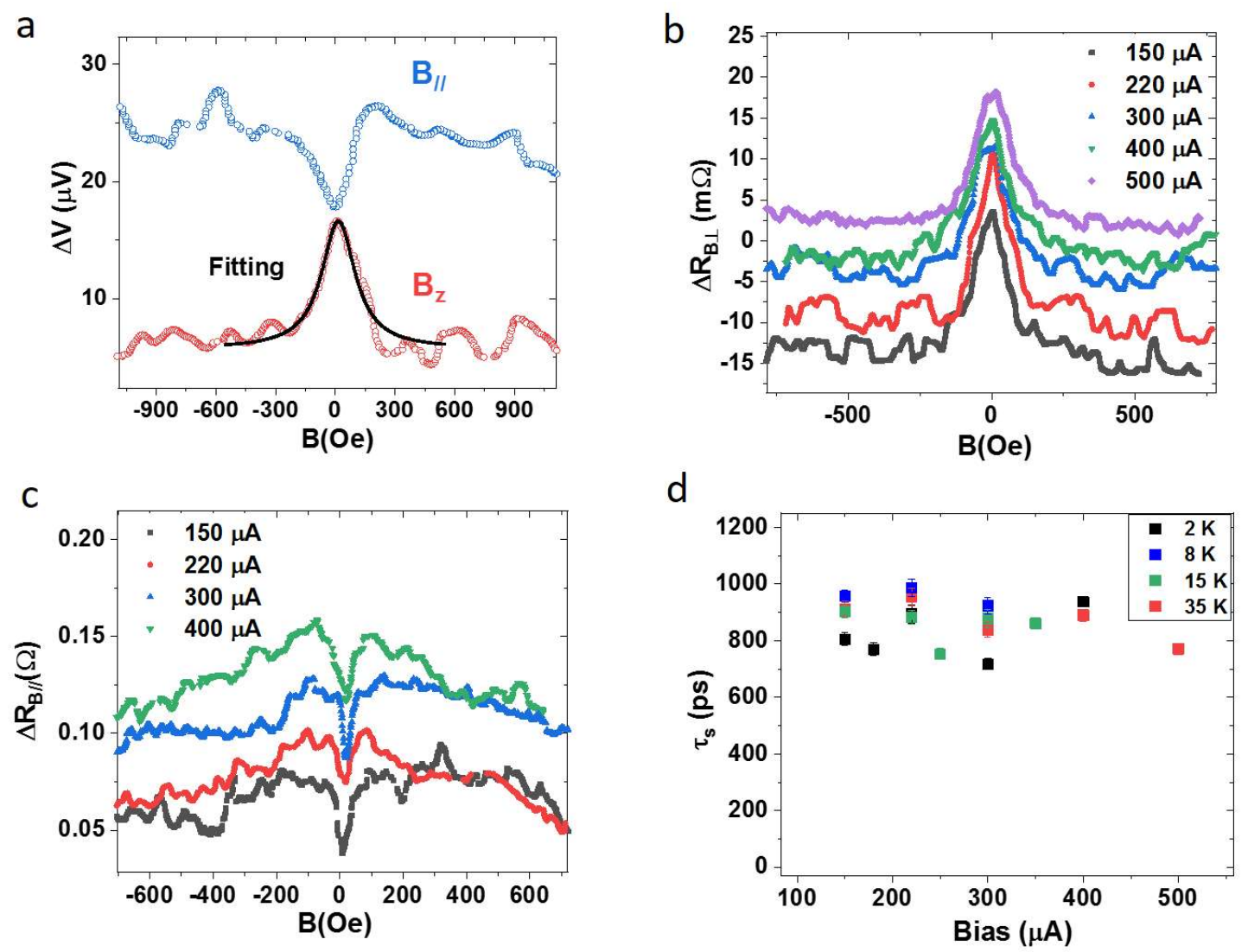

Figure 3. (a) 3T magnetoresistance under magnetic field perpendicular and parallel to the surface at $55 \mathrm{~K}$ with $\mathrm{AC} 300 \mu \mathrm{A}$. (b) Magnetoresistance measured with perpendicular magnetic field (Hanle signals) at $35 \mathrm{~K}$ with various $\mathrm{AC}$ current. (c) Magnetoresistance with magnetic field parallel to the surface (inverted Hanle signals) at $35 \mathrm{~K}$ with various $\mathrm{AC}$ current. (d) Extracted spin relaxation time as a function of bias at different temperatures. 

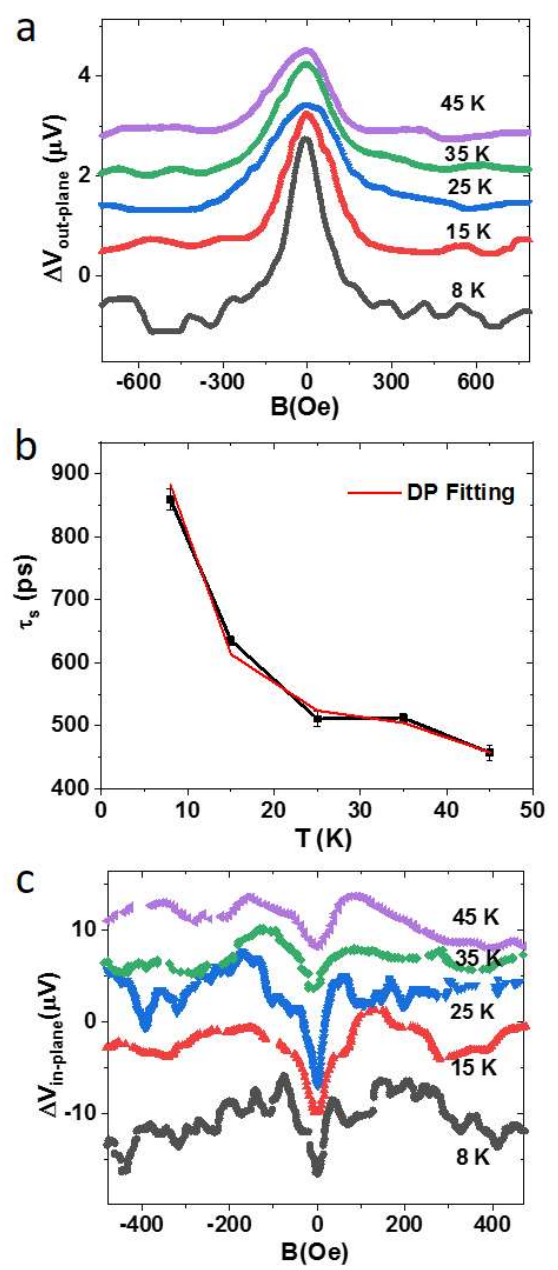

Figure 4. (a) Spin dependent voltage with perpendicular magnetic field at $200 \mu \mathrm{A}$ at different temperatures. (b) Extracted spin relaxation time with Lorentz fitting as a function of temperature. The red curve is the fit with DP spin relaxation mechanism as a function of temperature. (c) Spin dependent voltage with magnetic field parallel to the surface at different temperatures. 